\title{
Histamine phosphate has a cumulative effect when inhaled at five minute intervals
}

\author{
C TREMBLAY, I LEMIRE, H GHEZZO, L PINEAU, RR MARTIN, A CARTIER, JL MALO \\ From the Department of Chest Medicine, Hôpital du Sacré-Coeur, Montreal, Quebec, Canada
}

ABSTRACT As the duration of bronchoconstriction induced by inhaled histamine phosphate is greater than five minutes, a study was carried out to determine whether this leads to a cumulative effect when histamine is inhaled at five minute intervals as in standardised procedures. Fourteen clinically stable adult asthmatic subjects were studied. In the first part of the study (the noncumulative stage) they inhaled doubling concentrations of histamine until appreciable bronchoconstriction occurred (changes close to $50 \%$ in lung resistance for seven subjects and $15 \%$ in forced expiratory volume in one second for seven others). After functional recovery the last concentration of histamine was nebulised on two more occasions, allowing for functional recovery after each nebulisation. In the second part of the study (the cumulative stage) subjects inhaled, depending on their responsiveness, three to eight consecutive doses of the histamine concentration last administered in the non-cumulative stage, these doses being administered at five minute intervals, without recovery. The cumulative effect was assessed by linear regression analysis of the changes in the specific functional indices, all the values obtained during the non-cumulative stage being given the score 0 and those obtained during the cumulative step scores of 1,2 , etc. In all but one instance significant $(p<0.01)$ correlations were obtained and the slopes were positive, thus showing a cumulative effect. It is concluded that histamine has a cumulative bronchoconstrictor effect if inhaled at five minute intervals once appreciable bronchoconstriction has been reached.

Inhalation of histamine phosphate is often used to confirm the diagnosis of asthma..$^{1-3}$ According to proposed standardised procedures, ${ }^{34}$ doubling concentrations of histamine phosphate are inhaled at five minute intervals until the forced expiratory volume in one second $\left(\mathrm{FEV}_{1}\right)$ falls by $20 \%$ or more. The concentration giving a $20 \%$ fall in $\mathrm{FEV}_{1}\left(\mathrm{PC}_{20}\right)$ is interpolated on the dose-response curve.

Studies of the time course of bronchoconstriction induced by inhaled histamine have shown that functional recovery takes longer than five minutes. ${ }^{56}$ This suggests that inhaled histamine could have a cumulative effect when inhaled at five minute intervals. Juniper et $\mathrm{al}^{7}$ were unable to detect such a cumulative effect by studying $\mathrm{PC}_{20}$ on two occasions, firstly by nebulising progressively doubling concentrations of histamine, as in the commonly accepted

Address for reprint requests: Dr Jean-Luc Malo, Department of Chest Medicine, Hôpital du Sacré-Coeur, Montreal, Quebec, Canada, H4J $1 \mathrm{C} 5$.

Accepted 4 June 1984 methods, and secondly by administering on a separate occasion one dose of the concentration last used in reaching $\mathrm{PC}_{20}$ in the progressive method. ${ }^{7}$

We wished to determine whether histamine could have a cumulative effect by comparing the serial changes in bronchoconstriction when histamine was given with time for functional recovery between each nebulisation and the changes occurring when it was given at five minute intervals without recovery.

\section{Methods}

SUBJECTS

Fourteen adult asthmatic subjects ${ }^{8}$ were included in this study. There were five men and nine women, all of whom were clinically stable (table 1 ). All had avoided exposure to relevant allergens (except for house dust) for at least two months before the study. Regular treatment was interrupted for the visit as recommended by the special committee of the American Academy of Allergy ${ }^{4}$ - that is, treatment with long acting theophylline derivatives was inter- 
Table 1 Baseline anthropometric, clinical, and physiological data

\begin{tabular}{|c|c|c|c|c|c|c|c|c|}
\hline No & Sex & $\begin{array}{l}\text { Age } \\
(y)\end{array}$ & $\begin{array}{l}\text { Height } \\
\text { (cm) }\end{array}$ & $\begin{array}{l}F E V_{1} \\
(\% p)\end{array}$ & $\begin{array}{l}F E F_{25-75 \%} \\
(\% p)\end{array}$ & $\begin{array}{l}P C_{4} a s G L \\
(\mathrm{mg} / \mathrm{ml})\end{array}$ & $\begin{array}{l}P C_{15 F E V_{1}} \\
(\mathrm{mg} / \mathrm{ml})\end{array}$ & $\begin{array}{l}\text { Usual } \\
\text { treatment }\end{array}$ \\
\hline $\begin{array}{l}1 \\
2 \\
3 \\
4 \\
5 \\
6 \\
7 \\
8 \\
9 \\
10 \\
11 \\
12 \\
13 \\
14 \\
\text { Mean } \\
\text { SD }\end{array}$ & $\begin{array}{l}\mathbf{F} \\
\mathbf{F} \\
\mathbf{F} \\
\mathbf{M} \\
\mathbf{F} \\
\mathbf{M} \\
\mathbf{F} \\
\mathbf{M} \\
\mathbf{F} \\
\mathbf{M} \\
\mathbf{M} \\
\mathbf{F} \\
\mathbf{F} \\
\mathbf{F}\end{array}$ & $\begin{array}{l}57 \\
44 \\
36 \\
45 \\
38 \\
55 \\
27 \\
21 \\
24 \\
34 \\
54 \\
24 \\
53 \\
50 \\
40 \cdot 1 \\
12 \cdot 8\end{array}$ & $\begin{array}{c}160 \\
162 \\
162 \\
170 \\
178 \\
168 \\
168 \\
163 \\
152 \\
177 \\
175 \\
155 \\
159 \\
163 \\
165 \cdot 1 \\
7.9\end{array}$ & $\begin{array}{c}99 \\
73 \\
78 \\
75 \\
120 \\
65 \\
103 \\
101 \\
84 \\
107 \\
64 \\
99 \\
106 \\
79 \\
89 \cdot 5 \\
17.6\end{array}$ & $\begin{array}{l}46 \\
36 \\
35 \\
34 \\
81 \\
35 \\
56 \\
67 \\
60 \\
61 \\
29 \\
98 \\
92 \\
35 \\
54 \cdot 6 \\
23 \cdot 0\end{array}$ & $\begin{array}{l}0.44 \\
0.05 \\
0.09 \\
0.06 \\
2.05 \\
0.09 \\
1.26\end{array}$ & $\begin{array}{l}0.22 \\
4 \cdot 0 \\
3.7 \\
0.24 \\
0.35 \\
1.0 \\
0.06 \\
0.21 \\
10.8\end{array}$ & $\begin{array}{l}\text { B, Beclo } \\
\text { B, Beclo, IB } \\
\text { B, Beclo } \\
\text { B } \\
\text { B prn } \\
\text { B, Beclo } \\
\text { B prn } \\
\text { B prn } \\
\text { B prn } \\
\text { B prn } \\
\text { B, Beclo } \\
\text { B, Beclo } \\
\text { B, Beclo } \\
\text { B, Beclo, IB }\end{array}$ \\
\hline
\end{tabular}

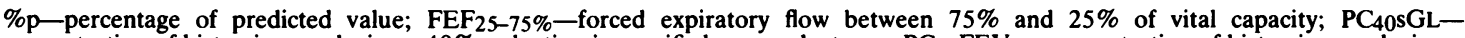
concentration of histamine producing a $40 \%$ reduction in specific lung conductance; $\mathrm{PC}_{15} \mathrm{FEV} \mathrm{F}_{1}$-concentration of histamine producing a $15 \%$ fall in $\mathrm{FEV}_{1}$; B-oral theophylline derivative or inhaled $\beta_{2}$ adrenergic agent, or both; Beclo-inhaled beclomethasone; IB-inhaled ipratropium bromide; prn-only if needed (not daily).

rupted 12 hours and inhaled $\beta_{2}$ adrenergic agents eight hours before the study. Ipratropium bromide inhalation was interrupted eight hours before; treatment with beclomethasone was not modified.

\section{PROTOCOL}

Subjects were studied on one occasion. After a rest of 10-15 minutes subjects were asked to produce forced expiratory manoeuvres, from which FEV , $_{1}$ forced vital capacity (FVC), and maximum forced expiratory flow rates $\left(\mathrm{FEF}_{25-75 \%}\right)$ were obtained according to the criteria of the American Thoracic Society, ${ }^{9}$ the best of two reproducible $( \pm 5 \%) \mathrm{FEV}_{1}$ and FVC manoeuvres being used and FEF $_{25-75 \%}$ being derived from the curve with the best $\mathrm{FEV}_{1}$ and FVC. Then in the non-cumulative stage of the protocol subjects were asked to inhale progressively doubling concentrations of histamine at five minute intervals with longer intervals to permit functional recovery between each dose whenever necessary (changes in lung resistance $(R L)>20 \%$ or $\mathrm{FEV}_{1}>$ $5 \%$ ) until RL or FEV, changed by nearly $50 \%$ and $15 \%$ respectively from the values obtained after inhalation of phosphate buffered saline (PBS). Such changes appeared sufficient for this. If more severe bronchoconstriction had been reached in the noncumulative stage, consecutive nebulisations of histamine would have been hazardous in the cumulative stage (see below). After a first functional recovery ( $\pm 5 \%$ for $F E V_{1}$ and $\pm 20 \%$ for $R L$ compared with the post PBS measurements), the last concentration of histamine was inhaled again and then time was allowed for functional recovery. This was repeated a third time, and again the subjects were allowed to recover fully. (One subject received only two nebulisations owing to an excessively long period of recovery between each nebulisation.)

To evaluate the possible cumulative effect, the last concentration of histamine used in the noncumulative challenge was then nebulised at five minute intervals, with no functional recovery allowed, for three to eight doses, depending on the severity of each subject's airways obstruction and symptoms.

\section{MEASUREMENTS}

Solutions of histamine were inhaled through the Wright nebuliser (output $0.14 \mathrm{ml} / \mathrm{min}$; flow rate $8.5 \mathrm{I} / \mathrm{min}$; mean mass diameter of inhaled particles $\left.1 \cdot 3 \mu \mathrm{m}^{10}\right)$. Histamine was inhaled by the subjects breathing at ordinary tidal volume, using an orofacial mask for two minutes. Baseline spirometric measurements were made with a Collins 9 litre water spirometer.

In seven subjects (1-7), bronchoconstriction was assessed by measurement of RL and thoracic gas volume (TGV), from which specific lung conductance (sGL) was derived. RL was monitored every minute up to five minutes in both the cumulative and the non-cumulative phases of the study. It was also assessed approximately every five minutes thereafter until recovery in the non-cumulative part of the study. TGV was measured two to three minutes after the end of nebulisation and every five minutes thereafter until recovery, in the non-cumulative stage.

In the seven other subjects (8-14) the response was assessed by measuring $\mathrm{FEV}_{1}$ with the Collins spirometer. In the non-cumulative stage the FEV was measured 30,90 , and 180 seconds after the end of each nebulisation and every three minutes thereafter until recovery. In the cumulative part of the 
study it was measured 30,90 , and 180 seconds after the end of each nebulisation.

RL and TGV were measured in a flow displacement body plethysmograph. " Pleural pressure was estimated from a $10 \mathrm{~cm}$ oesophageal balloon ${ }^{12}$ connected to a Validyne differential pressure transducer via a $100 \mathrm{~cm}$ polyethylene catheter. The other side of the transducer was connected to an oral pressure tap to estimate transpulmonary pressure. $R \mathbf{L}$ was measured as the ratio of change in transpulmonary pressure to change in buccal flow during tidal breathing. TGV was assessed by asking the subjects to pant three or four times in two to four seconds while a shutter was closed at the end of a normal expiration.

\section{ANALYSIS OF RESULTS}

At each histamine concentration the percentage change in the functional indices was calculated from the following formula: highest RL posthistamine highest RL after PBS/highest RL after PBS in the case of measurements obtained for RL. For $\mathrm{SGL}$ and $\mathrm{FEV}_{1}$ the formula was: lowest value after histamine - lowest value after PBS/lowest value after PBS. Dose response curves were drawn on a semi logarithmic graph, the non-cumulative histamine

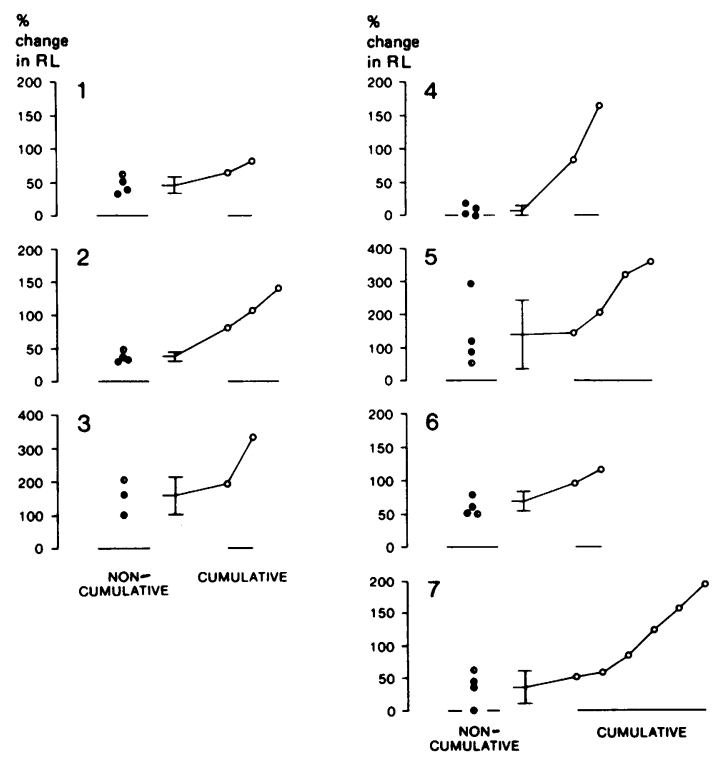

Fig 1 Percentage change in lung resistance $(R L)$ at each inhalation of histamine (horizontal axis) in non-culmulative $(O)$ and cumulative $(O)$ challenges. One point $(O)$ may be regarded as the last point of the non-culmulative stage or the first point of the cumulative stage as there was functional recovery between the two stages. The means and standard deviations for the inhalations of the non-cumulative stage are shown.

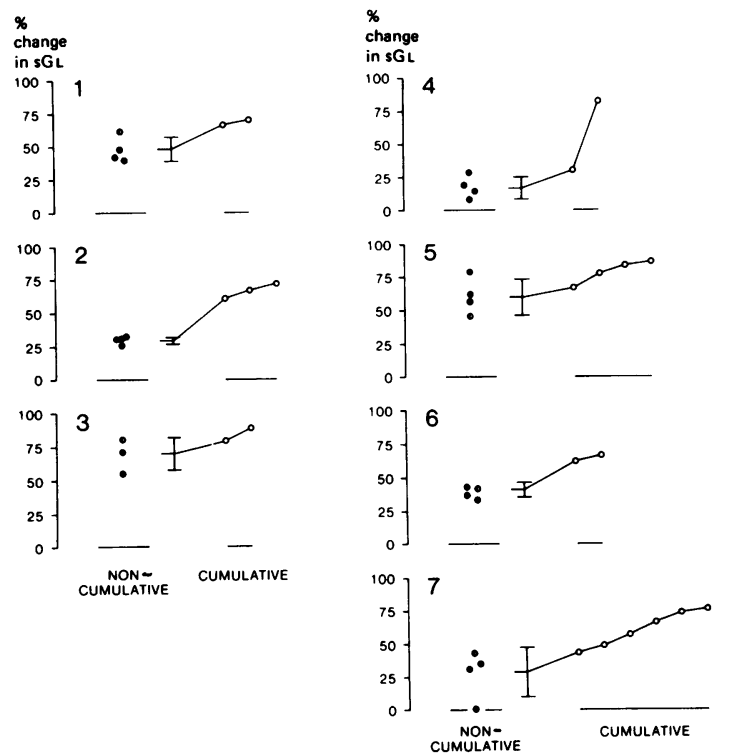

Fig 2 Percentage change in specific lung conductance (sGL) at each histamine dose in non-cumulative and cumulative challenges (symbols as in fig 1). Data are from the challenges shown in figure 1 .

concentration on the abscissa, and the percentage change in $F_{E V}, R L$, or SGL on the ordinate. The concentrations causing a $15 \%$ change in FEV $\left(\mathrm{PC}_{15} \mathrm{FEV}_{1}\right)$ and a $40 \%$ change in $\mathrm{SGL}\left(\mathrm{PC}_{40} \mathrm{sGL}\right)$

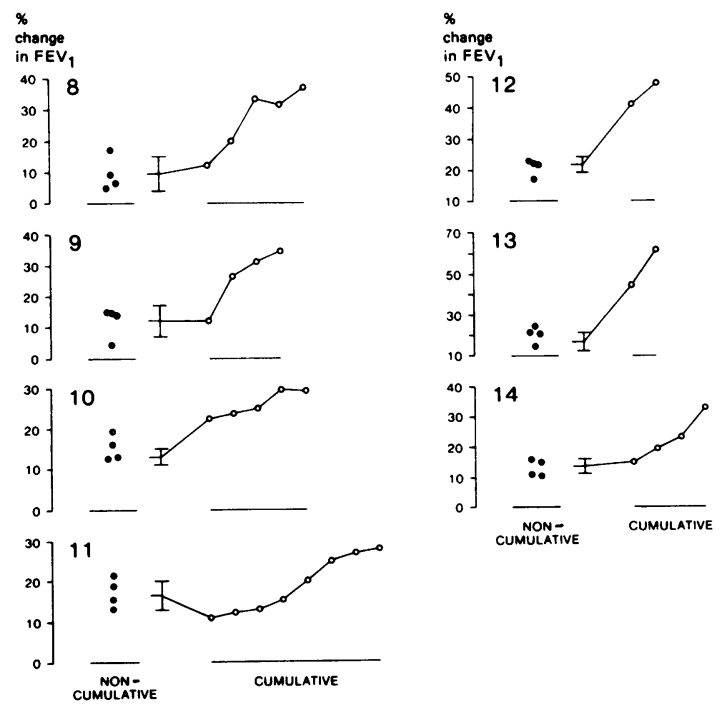

Fig 3 Percentage change in FEV at each histamine inhalation in non-cumulative and cumulative challenges in seven further asthmatic subjects (8-14). 
Table 2 Results of the regression analysis of percentage change in functional index and histamine concentration

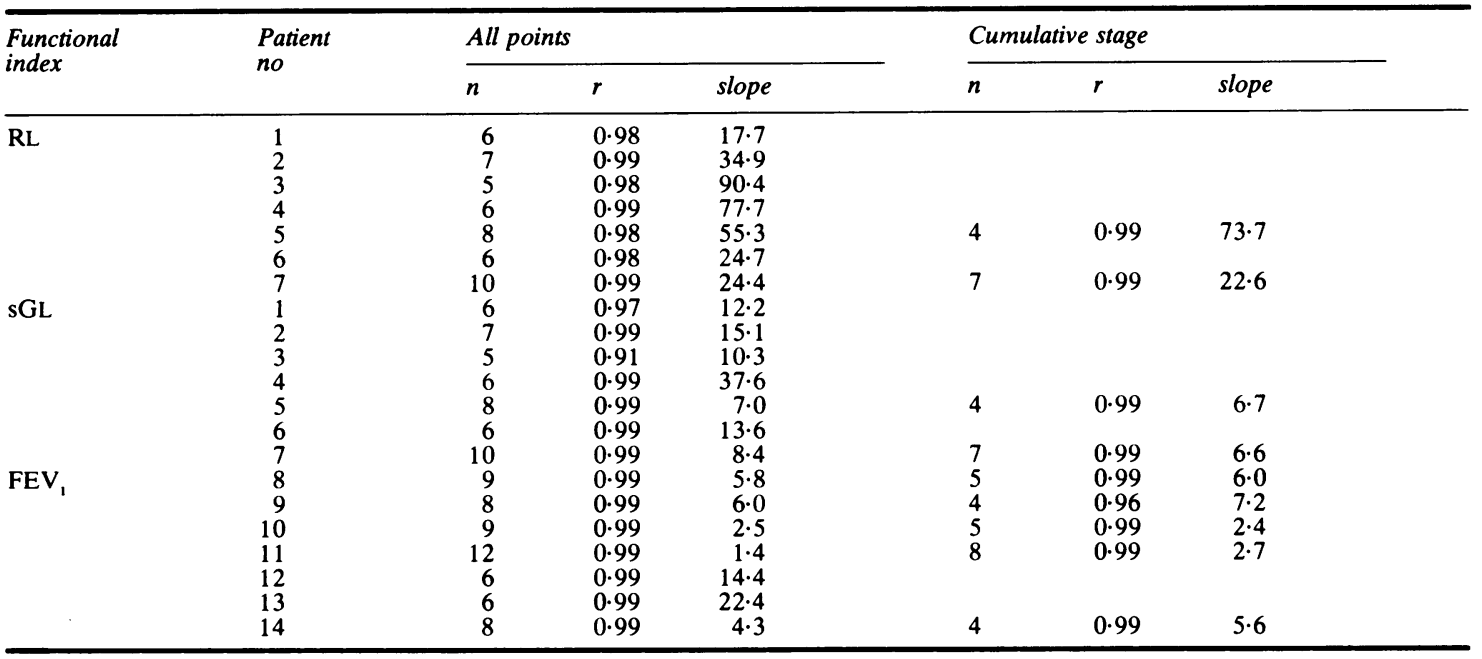

In the regression analysis including all points all values obtained during the non-cumulative stage were given a score of 0 and those during the cumulative stage were scored as 1,2 , etc. Only regression curves with four and more points were kept for analysis at the cumulative stage. RL-lung resistance; $s G L-$ specific lung conductance; $n-$ number of points; $r$-correlation coefficient; slope is expressed as \% change/score.

were interpolated on the dose-response curve. Reference values for $\mathrm{FEV}_{1}, \mathrm{FEV}_{1} / \mathrm{FVC}$, and $\mathrm{FEF}_{25-75 \%}$ were obtained from Knudson et al. ${ }^{13}$

\section{STATISTICAL ANALYSIS}

The cumulative effect was measured by linear regression analysis on the changes in the value of the functional parameters $\left(\mathrm{RL}, \mathrm{sGL}, \mathrm{FEV} \mathrm{V}_{1}\right)$. All the non-cumulative results were given the score zero and the cumulative results were given scores of 1,2 , etc. In all the patients except No 3 this gave four values at point 0 (since the first point of the cumulative step can also be interpreted as the last value of the non-cumulative stage, as there was functional recovery between the two stages) and one value at each consecutive period of the cumulative step with a different length for each subject. The regression line thus calculated will pass at point 0 through a point close to the mean value, and the points derived from the cumulative stage will determine whether the slope is positive, indicating a cumulative effect, or whether it is non-significant, reflecting only random variations. In the subjects for whom more than three points were obtained at the cumulative level a regression line was also computed through these points only. Concordance between the two slopes confirmed their representativity. Student's unpaired $t$ test was used for comparison of recovery intervals.

\section{Results}

Table 1 shows that six and 11 individuals respec- tively had baseline $\mathrm{FEV}_{1}$ or $\mathrm{FEF}_{25-75 \%}$ values below $80 \%$ of predicted. $\mathrm{PC}_{15} \mathrm{FEV}_{1}$ varied from 0.05 to $4 \mathrm{mg} / \mathrm{ml}$ and $\mathrm{PC}_{40} \mathrm{sGL}$ from 0.05 to $2.1 \mathrm{mg} / \mathrm{ml}$. The mean (SD) recovery intervals in the non-cumulative stage were $28.3(16 \cdot 6)$ and $15.6(8.2)$ minutes for RL and FEV 1 respectively $(t=3 \cdot 26, \mathrm{p}<0.01)$. These intervals were greater than five minutes in all instances except one.

Figures 1 and 2 show for each individual the percentage change in RL and SGL after each nebulisation of the non-cumulative and cumulative challenges in subjects 1-7. Figure 3 gives the same information for $\mathrm{FEV}_{1}$ for the seven other subjects (8-14). The ranges of percentage change in $R L$, sGL, and $F E V_{1}$ obtained at the first nebulisation of the non-cumulative stage were $16 \cdot 7-291 \cdot 5,18.4$ $77 \cdot 7$, and $15 \cdot 1-22 \cdot 4$. It can be seen that the changes obtained in the cumulative challenge steadily increase and reach values beyond 2 SD of the mean of the four values of the non-cumulative step in all instances for RL and $F E V_{1}$ and in six out of seven instances for sGL. Table 2 gives the corresponding statistical analysis using the regression analysis. All the non-cumulative values were given the score 0 and the cumulative values scores 1,2 , etc. The regression analysis showed highly significant $(\mathrm{p}<$ 0.01 ) correlation coefficients (except for subject 3 in the case of $s G L$ ) and positive slopes, thus confirming the cumulative effect. In the subjects for whom more than three points were determined in the cumulative part of the study the slopes were similar to those calculated with all the points (non- 
cumulative and cumulative steps), thus confirming the representativity of these slopes.

\section{Discussion}

Our study shows that histamine has a cumulative bronchoconstriction effect when inhaled at five minute intervals once significant bronchoconstriction has been reached. These results could have been predicted, on the grounds that the time course of the bronchoconstriction secondary to histamine is generally beyond five minutes. ${ }^{56} \mathrm{Juniper}$ et $\mathrm{al}^{7}$ found that $\mathrm{PC}_{20} \mathrm{FEV}_{1}$ obtained from a standard doseresponse curve was not significantly different from the value extrapolated by giving only the last concentration used in the standard dose-response curve. This suggested that histamine had no cumulative effect when the $\mathrm{PC}_{20} \mathrm{FEV}_{1}$ is the index used to assess the response. Even if $\mathrm{PC}_{20} \mathrm{FEV}_{1}$ does not seem to be affected significantly by consecutive nebulisations of histamine at five minute intervals, this does not exclude the possibility that histamine has a cumulative effect when other means of assessing the bronchoconstrictor response are used. In our study we assessed the percentage change of the physiological parameter from the post PBS value after each histamine inhalation. $\mathrm{PC}_{20} \mathrm{FEV}_{1}$ might not be affected by the cumulative effect of histamine as it is an index which lacks sensitivity by comparison with other indices used to assess the bronchoconstrictor response, ${ }^{14}$ whose reproducibility between two assessments is $1 \cdot 0-1 \cdot 6^{14}$ single twofold concentration differences. This range of reproducibility may preclude a satisfactory assessment of any cumulative effect. Juniper's results and ours can be reconciled in the following way: histamine has a cumulative effect when inhaled at five minute intervals, but this does not significantly affect $\mathrm{PC}_{20} \mathrm{FEV}_{1}$ results.

Even if forced expiratory manoeuvres can induce bronchodilation in the presence of induced bronchoconstriction, ${ }^{16-19}$ it is interesting that the cumulative effect could be detected with FEV in the same way as with indices not requiring maximal inspiratory manoeuvres. This suggests therefore that the bronchodilator effect is overcome by the cumulative bronchoconstrictor effect.

It is unlikely that the changes in functional indices after each nebulisation at the non-cumulative stage were influenced by the previous histamine inhalation. Firstly, functional recovery was observed between each nebulisation causing significant bronchoconstriction, even before the one selected for consecutive nebulisations at the non-cumulative and cumulative steps. Secondly, it is known that histamine does not induce tachyphylaxis in man when inhaled in a consecutive way with functional recov- ery between each test. ${ }^{2021}$

The cumulative effect of histamine when inhaled at five minute intervals could be explained in two ways. A pharmacological cumulative effect could be induced as each concentration is added to the previous one without the latter being completely in metabolised. The other cumulative effect is linked $\vec{\circ}$ with airway calibre. Once significant bronchoconstriction is induced, any further decrease in airway $\vec{\omega}$ calibre causes an even greater change in airflow obstruction, as resistance is inversely proportional to $\overrightarrow{\vec{x}}$ the fourth power of the radius in laminar flow conditions. ${ }^{2}$

Should dose-response curves for histamine be $\vec{N}$ expressed on a cumulative or on a non-cumulative $\frac{\vec{\sigma}}{\sigma}$ scale? Even if our study shows that histamine has a 0 cumulative effect when inhaled at five minute intervals, we have reason to suspect that the degree of $\vec{\nabla}$ this cumulative effect is not important. Firstly, the value of $\mathrm{PC}_{20}$, which is most often expressed on a non-cumulative scale, ${ }^{3}$ may vary by a single twofold concentration even in the case of a full pharmacological cumulative effect and is not significantly $\vec{\theta}$ influenced by this cumulative effect. ${ }^{7}$ In the case of a $\stackrel{\infty}{\oplus}$ full pharmacological additive effect all the concentrations from 0.03 to $16 \mathrm{mg} / \mathrm{ml}$ would total $32 \mathrm{mg} /$ $\mathrm{ml}$ of inhaled histamine if the results were expressed on a cumulative scale. The difference between $16 \mathrm{mg} / \mathrm{ml}$ and $32 \mathrm{mg} / \mathrm{ml}$ is within the reproducibility range of the test. ${ }^{14} 15$ Secondly, the physiological cumulative effect mentioned above would apply only when significant bronchoconstriction is reached, as was the case with the protocol used in the present study. The possibility that a cumulative effect might be noticed at lower concentrations not causing significant bronchoconstriction remains to be explored. Thus the physiological cumulative effect might only be present once significant bronchoconstriction is reached and not for the complete scale of the dose-response curves. It has been proposed $^{4}$ that the results of provocative inhalation tests $\mathrm{O}$ for histamine should be expressed in a cumulative way. This, however, would assume a completely cumulative effect, which is unlikely for the reasons mentioned. To express histamine dose-response $\mathcal{N}$ curves in a cumulative way rather than on a non- $N$ cumulative scale seems imprecise. Other studies are N needed to quantify the degree of the cumulative effect.

We thank l'Association Pulmonaire du Québec, which supported this work. RRM is a scholar of the Conseil de Recherche en Santé du Québec. IL was a ō summer student of the Université de Montréal sup- $\overrightarrow{\mathbb{D}}$ ported by a grant from the Fonds de la Recherche en Santé du Québec. We also express our gratitude to 
the subjects who took part in this study; to Patricia Hudson, who revised the manuscript; and to Lyette Rochon for her secretarial assistance.

\section{References}

1 Orehek J, Gayrard P. Les tests de provocation bronchique non-spécifique dans l'asthme. Bull Eur Physiopathol Respir 1976;12:565-98.

2 Boushey HA, Holtzman MK, Sheller JR, Nadel JA. Bronchial hyperreactivity. Am Rev Respir Dis 1980; 121:389-413.

3 Hargreave FE, Ryan G, Thomson NC, et al. Bronchial responsiveness to histamine or methacholine in asthma: measurement and clinical significance. $J$ Allergy Clin Immunol 1981;68:347-55. :

4 Chai H, Farr RS, Froehlich LA, et al. Standardization of bronchial inhalation chellenge procedures. J Allergy Clin Immunol 1975;56:323-7.

5 Mathé AA, Hedgvist P, Holmgren A, Svanborg N. Bronchial hyperreactivity to prostaglandin F2 alpha and histamine in patients with asthma. $\mathrm{Br}$ Med J 1973;i: 193-6.

6 Cartier A, Malo JL, Bégin P, Sestier M, Martin RR. Time course of the bronchoconstriction induced by inhaled histamine and methacholine. J Appl Physiol 1983;54:821-6.

7 Juniper EF, Frith PA, Dunnett C, Cockcroft DW, Hargreave FE. Reproducibility and comparison of responses to inhaled histamine and methacholine. Thorax 1978; 33: 705-10.

8 Committee of Diagnostic Standards for Nontuberculous Respiratory Diseases. Chronic bronchitis, asthma and pulmonary emphysema. A statement by the committee of diagnostic standards for nontuberculous respiratory diseases. $A m$ Rev Respir Dis 1962;85:762-8.

9 American Thoracic Society. Statement from Snowbird workshop on standarization of spirometry. Am Rev Respir Dis 1979;119:831-8.

10 Ryan G, Dolovich MB, Obminski G, et al. Standardization of inhalation provocation tests: influence of nebulizer output, particle size and method of inhalation. J Allergy Clin Immunol 1981;67:156-61.

11 Leith DE, Mead J. Principles of body plethysmography. In: Procedures for standarized measurements of lung mechanics. Bethesda, Maryland: National Heart and Lung Institute, 1974. (Distributed by the division of lung diseases.)

12 Milic-Emili J, Mead J, Turner JM, Glauser EM. Improved techniques for estimating pleural pressure from oesophageal balloons. J Appl Physiol 1964; 19:207-11.

13 Knudson RJ, Slatin RC, Lebowitz MD, Burrows B. The maximal expiratory flow-volume curves. Am Rev Respir Dis 1976;113:587-600.

14 Dehaut P, Rachiele A, Martin RR, Malo JL. Histamine dose-response curves in asthma: reproducibility and sensitivity of different indices to assess response. Thorax 1983;38:516-22.

15 Ryan G, Dolovich MB, Roberts RS, et al. Standardization of inhalation provocation tests: two techniques of aerosol generation and inhalation compared. $A m R e v$ Respir Dis 1981;123:195-9.

16 Nadel JA, Tierney DF. Effect of a previous deep inspiration on airway resistance in man. J Appl Physiol 1961;16:717-9.

17 Fish JE, Ankin MG, Kelly JF, Peterman VI. Regulation of bronchomotor tone by lung inflation in asthmatic and nonasthmatic subjects. J Appl Physiol 1981; 50: $1079-86$.

18 Beaupré A, Orehek J. Factors influencing the bronchodilator effect of a deep inspiration in asthmatic patients with provoked bronchoconstriction. Thorax 1982;37:124-8.

19 Sestier M, Pineau L, Cartier A, Martin RR, Malo JL. Bronchial responsiveness to methacholine and effects of respiratory manoeuvres. J Appl Physiol 1984; 56: $122-8$.

20 Ruffin RE, Alpers JH, Crockett AJ, Hamilton R. Repeated histamine inhalation tests in asthmatic patients. J Allergy Clin Immunol 1981;67:285-9.

21 Lemire I, Cartier A, Malo JL, Pineau L, Ghezzo H, Martin RR. Effect of sodium cromoglycate on histamine inhalation tests. J Allergy Clin Immunol 1984;73:234-9. 